\title{
The Fixed Point Theory and the Existence of the Periodic Solution on a Nonlinear Differential Equation
}

\author{
Ni Hua \\ Faculty of Science, Jiangsu University, 301 Xuefu Road, Zhenjiang, Jiangsu 212013, China \\ Correspondence should be addressed to Ni Hua; nihua979@126.com
}

Received 3 June 2018; Revised 12 July 2018; Accepted 17 July 2018; Published 1 August 2018

Academic Editor: Mehmet Sezer

Copyright (c) $2018 \mathrm{Ni}$ Hua. This is an open access article distributed under the Creative Commons Attribution License, which permits unrestricted use, distribution, and reproduction in any medium, provided the original work is properly cited.

This paper deals with a nonlinear differential equation, by using the fixed point theory. The existence of the periodic solution of the nonlinear differential equation is obtained; these results are new.

\section{Introduction}

The nonlinear Abel-type first-order differential equation

$$
\frac{d x}{d t}=a(t) x^{3}+b(t) x^{2}+c(t) x+d(t)
$$

plays an important role in many physical and technical applications; because of its importance, many scholars have studied it [1-7].

Recently, A. Cima, A. Gasull, and F. Manosas [8] gave the maximum number of polynomial solutions of some integrable polynomial Abel differential equations; Jaume Giné Claudia and Valls [9] studied the center problem for Abel polynomial differential equations of second kind; Jianfeng Huang and Haihua Liang [10] were devoted to the investigation of Abel equation by means of Lagrange interpolation formula; they gave a criterion to estimate the number of limit cycles of the Abel's equations; Berna Bülbül and Mehmet Sezer [11] introduced a numerical power series algorithm which is based on the improved Taylor matrix method for the approximate solution of Abel-type differential equations; $\mathrm{Ni}$ et al. [12] discussed the existence and stability of the periodic solutions of (1) and obtained the sufficient conditions which guaranteed the existence and stability of the periodic solutions for (1) from a particular one.

In this paper, we consider the following more general nonlinear differential equation:

$$
\frac{d x}{d t}=f(t, x) x+g(t, x)
$$

firstly, we give two results about the existence and uniqueness of the periodic solution of (2) by using the fixed point theory; then, we use Lyapunov function method and discuss the stability of the periodic solution; further, we discuss (1) and get the existence of the periodic solution of (1); some new results are obtained.

\section{Preliminaries}

Lemma 1 (see [12]). Consider the following linear differential equation:

$$
\frac{d x}{d t}=a(t) x+b(t),
$$

where $a(t), b(t)$ are $\omega$-periodic continuous functions; if $\int_{0}^{\omega} a(t) d t \neq 0$, then for (3) there exists a unique $\omega$-periodic continuous solution $\eta(t)$, and $\eta(t)$ can be written as follows:

$$
\eta(t)= \begin{cases}\int_{-\infty}^{t} e^{\int_{s}^{t} a(\tau) d \tau} b(s) d s, & \int_{0}^{\omega} a(t) d t<0 \\ -\int_{t}^{+\infty} e^{\int_{s}^{t} a(\tau) d \tau} b(s) d s, & \int_{0}^{\omega} a(t) d t>0\end{cases}
$$

For the sake of convenience, suppose that $f(t)$ is an $\omega$ periodic continuous function on $R$; we denote

$$
\begin{aligned}
f_{M} & =\sup _{t \in[0, \omega]} f(t), \\
f_{L} & =\inf _{t \in[0, \omega]} f(t) .
\end{aligned}
$$


The rest of the paper is arranged as follows. We will study the existence and stability property of the periodic solution of system (2) in the next section and discuss the existence of the periodic solution of system (1) on Section 4. We end this paper with two examples.

\section{Periodic Solution on a Nonlinear Differential Equation}

Theorem 2. Consider (2); $f(t, x), g(t, x)$ are both $\omega$-periodic continuous functions on $t \in R$ and uniformly with respect to $x \in R$; suppose that the following conditions hold:

$$
\begin{aligned}
&\left(H_{1}\right) \quad f(t, x) \leq-\alpha<0 ; \\
&\left(H_{2}\right) \quad|f(t, x)-f(t, y)| \leq L_{1}|x-y|, \\
&|g(t, x)-g(t, y)| \leq L_{2}|x-y|, \quad L_{2}<\alpha ; \\
&\left(H_{3}\right) \quad \frac{\alpha L_{2}+L_{1} \sup _{t \in[0, \omega]}|g(t, 0)|-L_{2}^{2}}{\alpha\left(\alpha-L_{2}\right)}<1 ;
\end{aligned}
$$

then

(1) for (2) there exists a unique $\omega$-periodic continuous solution $\gamma(t)$, and

$$
|\gamma(t)| \leq \frac{1}{\alpha-L_{2}} \sup _{t \in[0, \omega]}|g(t, 0)|,
$$

(2) $\gamma(t)$ is uniformly asymptotic stable.

Proof. (1) Let

$$
\begin{aligned}
B & =\{\varphi(t)|\varphi \in C(R, R), \varphi(t+\omega)=\varphi(t),| \varphi(t) \mid \\
& \left.\leq \frac{1}{\alpha-L_{2}} \sup _{t \in[0, \omega]}|g(t, 0)|\right\} .
\end{aligned}
$$

Given any $\varphi(t) \in B$, the norm is defined as follows:

$$
\|\varphi\|=\sup _{t \in[0, \omega]}|\varphi(t)| ;
$$

thus $(B,\|\cdot\|)$ is a Banach space. Consider the following equation:

$$
\frac{d x}{d t}=f[t, \varphi(t)] x+g[t, \varphi(t)]
$$

by $\left(H_{1}\right)$, according to Lemma $1,(10)$ has a unique $\omega$-periodic continuous solution as follows:

$$
\eta(t)=\int_{-\infty}^{t} e^{\int_{s}^{t} f[\tau, \varphi(\tau)] d \tau} g[s, \varphi(s)] d s
$$

and

$$
\begin{aligned}
& |\eta(t)| \leq \int_{-\infty}^{t} e^{-\alpha(t-s)}|g[s, \varphi(s)]| d s \\
& \leq \int_{-\infty}^{t} e^{-\alpha(t-s)}\left(L_{2}|\varphi(s)|+|g(s, 0)|\right) d s \\
& \leq \frac{L_{2}|\varphi|_{M}+\sup _{t \in[0, \omega]}|g(t, 0)|}{\alpha} \\
& =\frac{L_{2}\left(1 /\left(\alpha-L_{2}\right)\right) \sup _{t \in[0, \omega]}|g(t, 0)|+\sup _{t \in[0, \omega]}|g(t, 0)|}{\alpha} \\
& =\frac{1}{\alpha-L_{2}} \sup _{t \in[0, \omega]}|g(t, 0)|
\end{aligned}
$$

Define a map as follows:

$$
(T \varphi)(t)=\eta(t)=\int_{-\infty}^{t} e^{\int_{s}^{t} f[\tau, \varphi(\tau)] d \tau} g[s, \varphi(s)] d s ;
$$

thus if given any $\varphi(t) \in B$, then $(T \varphi)(t) \in B$; hence $T: B \longrightarrow$ $B$; given any $\varphi(t), \psi(t) \in B$, we have

$$
\begin{aligned}
& |(T \varphi)(t)-(T \psi)(t)|=\mid \int_{-\infty}^{t} e^{\int_{s}^{t} f[\tau, \varphi(\tau)] d \tau} g[s, \varphi(s)] d s \\
& \quad-\int_{-\infty}^{t} e^{\int_{s}^{t} f[\tau, \psi(\tau)] d \tau} g[s, \psi(s)] d s \mid \\
& \quad=\mid \int_{-\infty}^{t} e^{\int_{s}^{t} f[\tau, \varphi(\tau)] d \tau}(g(s, \varphi(s))-g(s, \psi(s))) d s \\
& \quad+\int_{-\infty}^{t}\left(e^{\int_{s}^{t} f[\tau, \varphi(\tau)] d \tau}-e^{\int_{s}^{t} f[\tau, \psi(\tau)] d \tau}\right) g(s, \psi(s)) d s \mid \\
& =\mid \int_{-\infty}^{t} e^{\int_{s}^{t} f[\tau, \varphi(\tau)] d \tau}(g(s, \varphi(s))-g(s, \psi(s))) d s \\
& \quad+\int_{-\infty}^{t} e^{\xi}\left(\int_{s}^{t} f[\tau, \varphi(\tau)] d \tau-\int_{s}^{t} f[\tau, \psi(\tau)] d \tau\right) \\
& \quad \cdot g(s, \psi(s)) d s \mid ;
\end{aligned}
$$

here, $\xi$ is between $\int_{s}^{t} f[\tau, \varphi(\tau)] d \tau$ and $\int_{s}^{t} f[\tau, \psi(\tau)] d \tau$; thus $\xi \leq-\alpha(t-s)$, so we have

$$
\begin{aligned}
& |(T \varphi)(t)-(T \psi)(t)| \leq \int_{-\infty}^{t} e^{\int_{s}^{t} f[\tau, \varphi(\tau)] d \tau}|g(s, \varphi(s))-g(s, \psi(s))| d s \\
& \quad+\int_{-\infty}^{t} e^{\xi}\left|\int_{s}^{t} f[\tau, \varphi(\tau)] d \tau-\int_{s}^{t} f[\tau, \psi(\tau)] d \tau\right||g(s, \psi(s))| d s \mid \\
& \quad \leq \int_{-\infty}^{t} e^{\int_{s}^{t} f[\tau, \varphi(\tau)] d \tau}|g(s, \varphi(s))-g(s, \psi(s))| d s+\int_{-\infty}^{t} e^{\xi} \int_{s}^{t}|f[\tau, \varphi(\tau)]-f[\tau, \psi(\tau)]| d \tau|g(s, \psi(s))| d s \mid
\end{aligned}
$$




$$
\begin{aligned}
& \leq L_{2} \int_{-\infty}^{t} e^{-\alpha(t-s)}|\varphi(s)-\psi(s)| d s+L_{1} \int_{-\infty}^{t} e^{-\alpha(t-s)} \int_{s}^{t}|\varphi(\tau)-\psi(\tau)| d \tau|g(s, \psi(s))| d s \mid \\
& \leq L_{2} \int_{-\infty}^{t} e^{-\alpha(t-s)}|\varphi(s)-\psi(s)| d s+L_{1} \int_{-\infty}^{t} e^{-\alpha(t-s)} \int_{s}^{t}|\varphi(\tau)-\psi(\tau)| d \tau\left(L_{2}|\psi(s)|+|g(s, 0)|\right) d s \\
& =\left(L_{2} \int_{-\infty}^{t} e^{-\alpha(t-s)} d s+L_{1} \int_{-\infty}^{t} e^{-\alpha(t-s)}(t-s)\left(L_{2}|\psi(s)|+|g(s, 0)|\right) d s\right)\|\varphi-\psi\| \\
& \leq\left(\frac{L_{2}}{\alpha}+\frac{L_{1}\left(L_{2}|\psi|_{M}+\sup _{t \in[0, \omega]}|g(t, 0)|\right)}{\alpha^{2}}\right)\|\varphi-\psi\| \\
& =\left(\frac{L_{2}}{\alpha}+\frac{L_{1}\left(L_{2}\left(1 /\left(\alpha-L_{2}\right)\right) \sup _{t \in[0, \omega]}|g(t, 0)|+\sup _{t \in[0, \omega]}|g(t, 0)|\right)}{\alpha^{2}}\right)\|\varphi-\psi\| \\
& =\left(\frac{L_{2}}{\alpha}+\frac{\alpha L_{1} \sup _{t \in[0, \omega]}|g(t, 0)| /\left(\alpha-L_{2}\right)}{\alpha^{2}}\right)\|\varphi-\psi\|=\frac{\alpha L_{2}+L_{1} \sup _{t \in[0, \omega]}|g(t, 0)|-L_{2}^{2}}{\alpha\left(\alpha-L_{2}\right)}\|\varphi-\psi\|
\end{aligned}
$$

By $\left(H_{2}\right),\left(H_{3}\right)$, it follows that

$$
0<\frac{\alpha L_{2}+L_{1} \sup _{t \in[0, \omega]}|g(t, 0)|-L_{2}^{2}}{\alpha\left(\alpha-L_{2}\right)}<1
$$

by (16), $T$ is a compression mapping; according to the compression mapping principle, $T$ has a fixed point on $B$, the fixed point is the unique $\omega$-periodic continuous solution $\gamma(t)$ of (2), and $|\gamma(t)| \leq\left(1 /\left(\alpha-L_{2}\right)\right) \sup _{t \in[0, \omega]}|g(t, 0)|$.

(2) Define a Lyapunov function as follows:

$$
V(t, x(t))=(x(t)-\gamma(t))^{2},
$$

where $x(t)$ is the unique solution with the initial value $x\left(t_{0}\right)=$ $x_{0}$ of (2); differentiating both sides of (17) along the solution of (2), we get

$$
\begin{aligned}
& \frac{d V(t, x(t))}{d t}=2(x(t)-\gamma(t))\left[\frac{d x(t)}{d t}-\frac{d \gamma(t)}{d t}\right] \\
& \quad=2(x(t)-\gamma(t))[f(t, x(t)) x(t) \\
& \quad-f(t, \gamma(t)) \gamma(t)+(g(t, x(t))-g(t, \gamma(t)))] \\
& \quad=2(x(t)-\gamma(t))[f(t, x(t))(x(t)-\gamma(t)) \\
& \quad+(f(t, x(t))-f(t, \gamma(t))) \gamma(t) \\
& \quad+(g(t, x(t))-g(t, \gamma(t)))]=2 f(t, x)(x(t) \\
& -\gamma(t))^{2}+2(x(t)-\gamma(t))(f(t, x(t)) \\
& -f(t, \gamma(t))) \gamma(t)+2(x(t)-\gamma(t))(g(t, x(t)) \\
& -g(t, \gamma(t))) \leq 2 f(t, x)(x(t)-\gamma(t))^{2}+2 \mid x(t) \\
& -\gamma(t)|| f(t, x(t))-f(t, \gamma(t))|| \gamma(t)|+2| x(t)
\end{aligned}
$$

$$
\begin{aligned}
& -\gamma(t)|| g(t, x(t))-g(t, \gamma(t)) \mid \leq 2(x(t)-\gamma(t)) \\
& \cdot[f(t, x(t))(x(t)-\gamma(t)) \\
& \left.+L_{1}(x(t)-\gamma(t))|\gamma(t)|+L_{2}(x(t)-\gamma(t))\right] \\
& =2(x(t)-\gamma(t))^{2}\left[f(t, x(t))+L_{1}|\gamma(t)|+L_{2}\right] \\
& \leq 2(x(t)-\gamma(t))^{2}\left[-\alpha+\frac{L_{1}}{\alpha-L_{2}} \sup _{t \in[0, \omega]}|g(t, 0)|\right. \\
& \left.+L_{2}\right]=\frac{-\alpha^{2}+2 \alpha L_{2}-L_{2}^{2}+L_{1} \sup _{t \in[0, \omega]}|g(t, 0)|}{\alpha-L_{2}} \\
& \cdot 2(x(t)-\gamma(t))^{2}
\end{aligned}
$$

By $\left(H_{2}\right)$ and $\left(H_{3}\right)$, we have $\left(-\alpha^{2}+2 \alpha L_{2}-L_{2}^{2}+\right.$ $\left.L_{1} \sup _{t \in[0, \omega]}|g(t, 0)|\right) /\left(\alpha-L_{2}\right)<0$; there is a positive number $\delta$ such that $\left(-\alpha^{2}+2 \alpha L_{2}-L_{2}^{2}+L_{1} \sup _{t \in[0, \omega]}|g(t, 0)|\right) /\left(\alpha-L_{2}\right) \leq$ $-\delta<0$, and hence we have

$$
\frac{d V(t, x(t))}{d t} \leq-2 \delta(x(t)-\gamma(t))^{2} .
$$

Therefore, the periodic solution $\gamma(t)$ is uniformly asymptotic stable.

Theorem 3. Consider (2); $f(t, x), g(t, x)$ are both $\omega$-periodic continuous functions on $t \in R$ and uniformly, with respect to $x \in R$, suppose that the following conditions hold:

$$
\begin{aligned}
&\left(H_{1}\right) \quad f(t, x) \geq \alpha>0 ; \\
&\left(H_{2}\right) \quad|f(t, x)-f(t, y)| \leq L_{1}|x-y|, \\
&|g(t, x)-g(t, y)| \leq L_{2}|x-y|, \quad L_{2}<\alpha ; \\
&\left(H_{3}\right) \quad \frac{\alpha L_{2}+L_{1} \sup _{t \in[0, \omega]}|g(t, 0)|-L_{2}^{2}}{\alpha\left(\alpha-L_{2}\right)}<1 ;
\end{aligned}
$$


then

(1) for (2) there exists a unique w-periodic continuous solution $\gamma(t)$, and

$$
|\gamma(t)| \leq \frac{1}{\alpha-L_{2}} \sup _{t \in[0, \omega]}|g(t, 0)|,
$$

(2) $\gamma(t)$ is unstable.

Proof. (1) Let

$$
\begin{aligned}
B & =\{\varphi(t)|\varphi \in C(R, R), \varphi(t+\omega)=\varphi(t),| \varphi(t) \mid \\
& \left.\leq \frac{1}{\alpha-L_{2}} \sup _{t \in[0, \omega]}|g(t, 0)|\right\} .
\end{aligned}
$$

Given any $\varphi(t) \in B$, the norm is defined as follows:

$$
\|\varphi\|=\sup _{t \in[0, \omega]}|\varphi(t)|
$$

thus $(B,\|\cdot\|)$ is a Banach space. Consider the following equation:

$$
\frac{d x}{d t}=f[t, \varphi(t)] x+g[t, \varphi(t)]
$$

by $\left(H_{1}\right)$, according to Lemma $1,(24)$ has a unique $\omega$-periodic continuous solution as follows:

$$
\eta(t)=-\int_{t}^{+\infty} e^{\int_{s}^{t} f[\tau, \varphi(\tau)] d \tau} g[s, \varphi(s)] d s
$$

and

$$
\begin{aligned}
|\eta(t)| & \leq \int_{t}^{+\infty} e^{-\alpha(s-t)}|g[s, \varphi(s)]| d s \\
& \leq \int_{t}^{+\infty} e^{-\alpha(s-t)}\left(L_{2}|\varphi(s)|+|g(s, 0)|\right) d s
\end{aligned}
$$

$$
\begin{aligned}
& \leq \frac{L_{2}|\varphi|_{M}+\sup _{t \in[0, \omega]}|g(t, 0)|}{\alpha} \\
& =\frac{1}{\alpha-L_{2}} \sup _{t \in[0, \omega]}|g(t, 0)|
\end{aligned}
$$

Define a map as follows:

$$
(T \varphi)(t)=\eta(t)=-\int_{t}^{+\infty} e^{\int_{s}^{t} f[\tau, \varphi(\tau)] d \tau} g[s, \varphi(s)] d s ;
$$

thus if given any $\varphi(t) \in B$, then $(T \varphi)(t) \in B$; hence $T: B \longrightarrow$ $B$; given any $\varphi(t), \psi(t) \in B$, we have

$$
\begin{aligned}
& |(T \varphi)(t)-(T \psi)(t)| \\
& =\mid-\int_{t}^{+\infty} e^{\int_{s}^{t} f[\tau, \varphi(\tau)] d \tau} g[s, \varphi(s)] d s \\
& \quad+\int_{t}^{+\infty} e^{\int_{s}^{t} f[\tau, \psi(\tau)] d \tau} g[s, \psi(s)] d s \mid \\
& =\mid \int_{t}^{+\infty} e^{\int_{s}^{t} f[\tau, \varphi(\tau)] d \tau}(g(s, \varphi(s))-g(s, \psi(s))) d s \\
& \quad+\int_{t}^{+\infty}\left(e^{\int_{s}^{t} f[\tau, \varphi(\tau)] d \tau}-e^{\int_{s}^{t} f[\tau, \psi(\tau)] d \tau}\right) \\
& \cdot g(s, \psi(s)) d s \mid \\
& =\mid \int_{t}^{+\infty} e^{\int_{s}^{t} f[\tau, \varphi(\tau)] d \tau}(g(s, \varphi(s))-g(s, \psi(s))) d s \\
& \quad+\int_{t}^{+\infty} e^{\xi}\left(\int_{s}^{t} f[\tau, \varphi(\tau)] d \tau-\int_{s}^{t} f[\tau, \psi(\tau)] d \tau\right) \\
& \cdot g(s, \psi(s)) d s \mid ;
\end{aligned}
$$

here, $\xi$ is between $\int_{s}^{t} f[\tau, \varphi(\tau)] d \tau$ and $\int_{s}^{t} f[\tau, \psi(\tau)] d \tau$; thus $\xi \leq-\alpha(s-t)$, so we have

$$
\begin{aligned}
|(T \varphi)(t)-(T \psi)(t)| \leq & L_{2} \int_{t}^{+\infty} e^{-\alpha(t-s)}|\varphi(s)-\psi(s)| d s+L_{1} \int_{t}^{+\infty} e^{-\alpha(t-s)} \int_{s}^{t}|\varphi(\tau)-\psi(\tau)| d \tau|g(s, \psi(s))| d s \mid \\
\leq & L_{2} \int_{t}^{+\infty} e^{-\alpha(t-s)] d \tau}|\varphi(s)-\psi(s)| d s \\
& +L_{1} \int_{t}^{+\infty} e^{-\alpha(t-s)} \int_{s}^{t}|\varphi(\tau)-\psi(\tau)| d \tau\left(L_{2}|\psi(s)|+|g(s, 0)|\right) d s \\
= & \left(L_{2} \int_{t}^{+\infty} e^{-\alpha(t-s)} d s+L_{1} \int_{t}^{+\infty} e^{-\alpha(t-s)}(t-s)\left(L_{2}|\psi(s)|+|g(s, 0)|\right) d s\right)\|\varphi-\psi\| \\
\leq & \left(\frac{L_{2}}{\alpha}+\frac{L_{1}\left(L_{2}|\psi|_{M}+\sup _{t \in[0, \omega]}|g(t, 0)|\right)}{\alpha^{2}}\right)\|\varphi-\psi\|
\end{aligned}
$$




$$
\begin{aligned}
& =\left(\frac{L_{2}}{\alpha}+\frac{L_{1}\left(L_{2}\left(1 /\left(\alpha-L_{2}\right)\right) \sup _{t \in[0, \omega]}|g(t, 0)|+\sup _{t \in[0, \omega]}|g(t, 0)|\right)}{\alpha^{2}}\right)\|\varphi-\psi\| \\
& =\left(\frac{L_{2}}{\alpha}+\frac{\alpha L_{1} \sup _{t \in[0, \omega]}|g(t, 0)| /\left(\alpha-L_{2}\right)}{\alpha^{2}}\right)\|\varphi-\psi\|=\frac{\alpha L_{2}+L_{1} \sup _{t \in[0, \omega]}|g(t, 0)|-L_{2}^{2}}{\alpha\left(\alpha-L_{2}\right)}\|\varphi-\psi\|
\end{aligned}
$$

By $\left(H_{2}\right),\left(H_{3}\right)$, it follows that

$$
0<\frac{\alpha L_{2}+L_{1} \sup _{t \in[0, \omega]}|g(t, 0)|-L_{2}^{2}}{\alpha\left(\alpha-L_{2}\right)}<1
$$

by (30), $T$ is a compression mapping; according to the compression mapping principle, $T$ has a fixed point on $B$, the fixed point is the unique $\omega$-periodic continuous solution $\gamma(t)$ of (2), and $|\gamma(t)| \leq\left(1 /\left(\alpha-L_{2}\right)\right) \sup _{t \in[0, \omega]}|g(t, 0)|$.

(2) Define a Lyapunov function as follows:

$$
V(t, x(t))=(x(t)-\gamma(t))^{2},
$$

where $x(t)$ is the unique solution with the initial value $x\left(t_{0}\right)=$ $x_{0}$ of (2); differentiating both sides of (31) along the solution of (2), we get

$$
\begin{aligned}
& \frac{d V(t, x(t))}{d t}=2(x(t)-\gamma(t))\left[\frac{d x(t)}{d t}-\frac{d \gamma(t)}{d t}\right] \\
& =2(x(t)-\gamma(t))[f(t, x(t)) x(t) \\
& -f(t, \gamma(t)) \gamma(t)+(g(t, x(t))-g(t, \gamma(t)))] \\
& =2(x(t)-\gamma(t))[f(t, x(t))(x(t)-\gamma(t)) \\
& +(f(t, x(t))-f(t, \gamma(t))) \gamma(t) \\
& +(g(t, x(t))-g(t, \gamma(t)))]=2 f(t, x)(x(t) \\
& -\gamma(t))^{2}+2(x(t)-\gamma(t))(f(t, x(t)) \\
& -f(t, \gamma(t))) \gamma(t)+2(x(t)-\gamma(t))(g(t, x(t)) \\
& -g(t, \gamma(t))) \geq 2 f(t, x(t))(x(t)-\gamma(t))^{2} \\
& -2|x(t)-\gamma(t)||f(t, x(t))-f(t, \gamma(t))||\gamma(t)| \\
& -2|x(t)-\gamma(t)||g(t, x(t))-g(t, \gamma(t))| \\
& \geq 2(x(t)-\gamma(t))[f(t, x(t))(x(t)-\gamma(t)) \\
& \left.-L_{1}(x(t)-\gamma(t))|\gamma(t)|-L_{2}(x(t)-\gamma(t))\right] \\
& =2(x(t)-\gamma(t))^{2}\left[f(t, x(t))-L_{1}|\gamma(t)|-L_{2}\right]
\end{aligned}
$$

$$
\begin{aligned}
& \geq 2(x(t)-\gamma(t))^{2}\left[\alpha-\frac{L_{1}}{\alpha-L_{2}} \sup _{t \in[0, \omega]}|g(t, 0)|\right. \\
& \left.-L_{2}\right]=\frac{\alpha^{2}-2 \alpha L_{2}-L_{1} \sup _{t \in[0, \omega]}|g(t, 0)|+L_{2}^{2}}{\alpha-L_{2}} \\
& \cdot 2(x(t)-\gamma(t))^{2}
\end{aligned}
$$

By $\left(H_{2}\right)$ and $\left(H_{3}\right)$, we have $\left(\alpha^{2}-2 \alpha L_{2}-L_{1} \sup _{t \in[0, \omega]}|g(t, 0)|+\right.$ $\left.L_{2}^{2}\right) /\left(\alpha-L_{2}\right)>0$; there is a positive number $\delta$ such that $\left(\alpha^{2}-\right.$ $\left.2 \alpha L_{2}-L_{1} \sup _{t \in[0, \omega]}|g(t, 0)|+L_{2}^{2}\right) /\left(\alpha-L_{2}\right) \geq \delta>0$, and hence we have

$$
\frac{d V(t, x(t))}{d t} \geq 2 \delta(x(t)-\gamma(t))^{2}
$$

Therefore, the periodic solution $\gamma(t)$ is unstable.

Consider the following nonlinear differential equation:

$$
\frac{d x}{d t}=f(t, x) x+g(t)
$$

it is easy for us to draw the two corollaries about the existence and stability of the periodic solution on (34).

Corollary 4. Consider (34); $f(t, x)$ is $\omega$-periodic continuous functions on $t \in R$ and uniformly with respect to $x \in R, g(t)$ is an $\omega$-periodic continuous function on $t \in R$; suppose that the following conditions hold:

$$
\begin{array}{ll}
\left(H_{1}\right) & f(t, x) \leq-\alpha<0 ; \\
\left(H_{2}\right) & |f(t, x)-f(t, y)| \leq L|x-y| ; \\
\left(H_{3}\right) \quad L|g|_{M}<\alpha^{2}
\end{array}
$$

then

(1) for (34) there exists a unique $\omega$-periodic continuous solution $\gamma(t)$, and

$$
|\gamma(t)| \leq \frac{|g|_{M}}{\alpha},
$$

(2) $\gamma(t)$ is uniformly asymptotic stable.

Corollary 5. Consider (34); $f(t, x)$ is $\omega$-periodic continuous functions on $t \in R$ and uniformly with respect to $x \in R, g(t)$ is 
an $\omega$-periodic continuous function on $t \in R$, suppose that the following conditions hold:

$$
\begin{array}{ll}
\left(H_{1}\right) & f(t, x) \geq \alpha>0 \\
\left(H_{2}\right) & |f(t, x)-f(t, y)| \leq L|x-y| \\
\left(H_{3}\right) & L|g|_{M}<\alpha^{2}
\end{array}
$$

then

(1) for (34) there exists a unique w-periodic continuous solution $\gamma(t)$, and

$$
|\gamma(t)| \leq \frac{|g|_{M}}{\alpha}
$$

(2) $\gamma(t)$ is unstable.

\section{Periodic Solution on Abel's Differential Equation}

Theorem 6. Consider (1); $a(t), b(t), c(t), d(t)$ are all $\omega$-periodic continuous functions; suppose that the following conditions hold:

$$
\begin{array}{ll}
\left(H_{1}\right) & a(t)<0 \\
\left(H_{2}\right) & b^{2}(t)-3 a(t) c(t)<0 ; \\
\left(H_{3}\right) & -2 a_{L}|d|_{M}^{2}+\alpha|b|_{M}|d|_{M}-\alpha^{3}<0 ;
\end{array}
$$

here, $\alpha=\left(\left(b^{2}-4 a c\right) / 4 a\right)_{L}$; then

(1) for (1) there exists a unique w-periodic continuous solution $\gamma(t)$, and

$$
|\gamma(t)| \leq \frac{|d|_{M}}{\alpha}
$$

(2) $\gamma(t)$ is uniformly asymptotic stable.

Proof. (1) Define a set

$$
\begin{aligned}
B & =\{\varphi(t)|\varphi \in C(R, R), \varphi(t+\omega)=\varphi(t),| \varphi(t) \mid \\
& \left.\leq \frac{|d|_{M}}{\alpha}\right\} ;
\end{aligned}
$$

given any $\varphi(t) \in B$, the norm is defined as follows:

$$
\|\varphi\|=\sup _{t \in[0, \omega]}|\varphi(t)|
$$

thus $(B,\|\cdot\|)$ is a Banach space. Consider the following equation:

$$
\frac{d x}{d t}=\left[a(t) \varphi^{2}(t)+b(t) \varphi(t)+c(t)\right] x+d(t)
$$

By $\left(H_{1}\right)$ and $\left(H_{2}\right)$, we get that

$$
b^{2}(t)-4 a(t) c(t)<0
$$

and

$$
\begin{gathered}
a(t) \varphi^{2}(t)+b(t) \varphi(t)+c(t) \leq \frac{4 a(t) c(t)-b^{2}(t)}{4 a(t)} \\
\leq\left(\frac{4 a c-b^{2}}{4 a}\right)_{M}=-\left(\frac{b^{2}-4 a c}{4 a}\right)_{L}=-\alpha<0
\end{gathered}
$$

by Lemma $1,(43)$ has a unique continuous $\omega$-periodic solution as follows:

$$
\eta(t)=\int_{-\infty}^{t} e^{\int_{s}^{t}\left[a(\tau) \varphi^{2}(\tau)+b(\tau) \varphi(\tau)+c(\tau)\right] d \tau} d(s) d s
$$

by (46), we get

$$
|\eta(t)| \leq \frac{|d|_{M}}{\alpha}
$$

Define a map as follows:

$$
\begin{aligned}
(T \varphi)(t) & =\eta(t) \\
& =\int_{-\infty}^{t} e^{\int_{s}^{t}\left[a(\tau) \varphi^{2}(\tau)+b(\tau) \varphi(\tau)+c(\tau)\right] d \tau} d(s) d s
\end{aligned}
$$

thus if given any $\varphi(t) \in B$, then $(T \varphi)(t) \in B$; hence $T: B \longrightarrow$ $B$; given any $\varphi(t), \psi(t) \in B$, we have

$$
\begin{aligned}
|(T \varphi)(t)-(T \psi)(t)| & =\left|\int_{-\infty}^{t}\left\{e^{\int_{s}^{t}\left[a(\tau) \varphi^{2}(\tau)+b(\tau) \varphi(\tau)+c(\tau)\right] d \tau}-e^{\int_{s}^{t}\left[a(\tau) \psi^{2}(\tau)+b(\tau) \psi(\tau)+c(\tau)\right] d \tau}\right\} d(s) d s\right| \\
& =\left|\int_{-\infty}^{t} e^{\xi} \int_{s}^{t}\left\{a(\tau)\left[\varphi^{2}(\tau)-\psi^{2}(\tau)\right]+b(\tau)[\varphi(\tau)-\psi(\tau)]\right\} d \tau d(s) d s\right| \\
& =\left|\int_{-\infty}^{t} e^{\xi} \int_{s}^{t}[a(\tau)(\varphi(\tau)+\psi(\tau))+b(\tau)][\varphi(\tau)-\psi(\tau)] d \tau d(s) d s\right|
\end{aligned}
$$


here, $\xi$ is between $\int_{s}^{t}\left[a(\tau) \varphi^{2}(\tau)+b(\tau) \varphi(\tau)+c(\tau)\right] d \tau$ and $\int_{s}^{t}\left[a(\tau) \psi^{2}(\tau)+b(\tau) \psi(\tau)+c(\tau)\right] d \tau$; thus $\xi \leq-\alpha(t-s)$, so we have

$$
\begin{aligned}
& |(T \varphi)(t)-(T \psi)(t)| \\
& \quad \leq \int_{-\infty}^{t} e^{-\alpha(t-s)} \int_{s}^{t}|a(\tau)(\varphi(\tau)+\psi(\tau))+b(\tau)| d \tau \\
& \cdot|d(s)| d s\|\varphi-\psi\| \\
& \quad \leq \int_{-\infty}^{t} e^{-\alpha(t-s)} \int_{s}^{t}\left(2|a|_{M}|\varphi|_{M}+|b|_{M}\right) d \tau \\
& \cdot|d|_{M} d s\|\varphi-\psi\| \\
& =\int_{-\infty}^{t} e^{-\alpha(t-s)}\left(2|a|_{M}|\varphi|_{M}+|b|_{M}\right)(t-s) \\
& \cdot|d|_{M} d s\|\varphi-\psi\| \\
& =\frac{\left(2|a|_{M}|\varphi|_{M}+|b|_{M}\right)|d|_{M}}{\alpha^{2}}\|\varphi-\psi\| \\
& =\frac{\left(-2 a_{L}|\varphi|_{M}+|b|_{M}\right)|d|_{M}}{\alpha^{2}}\|\varphi-\psi\| \\
& =\frac{\left(-2 a_{L}\left(|d|_{M} / \alpha\right)+|b|_{M}\right)|d|_{M}}{\alpha^{2}}\|\varphi-\psi\|
\end{aligned}
$$

$$
\begin{aligned}
\frac{d V(t, x(t))}{d t} & =2(x(t)-\gamma(t))\left[\frac{d x(t)}{d t}-\frac{d \gamma(t)}{d t}\right] \\
& =2(x(t)-\gamma(t))\left[a(t)\left(x^{3}(t)-\gamma^{3}(t)\right)+b(t)\left(x^{2}(t)-\gamma^{2}(t)\right)+c(t)(x(t)-\gamma(t))\right] \\
& =2(x(t)-\gamma(t))^{2}\left[a(t)\left(x^{2}(t)+x(t) \gamma(t)+\gamma^{2}(t)\right)+b(t)(x(t)+\gamma(t))+c(t)\right] \\
& =2(x(t)-\gamma(t))^{2}\left[a(t) x^{2}(t)+(a(t) \gamma(t)+b(t)) x(t)+a(t) \gamma^{2}(t)+b(t) \gamma(t)+c(t)\right] \\
\leq & 2(x(t)-\gamma(t))^{2}\left[\frac{4 a(t)\left(a(t) \gamma^{2}(t)+b(t) \gamma(t)+c(t)\right)-(a(t) \gamma(t)+b(t))^{2}}{4 a(t)}\right] \\
& 2(x(t)-\gamma(t))^{2}\left[\frac{3 a^{2}(t) \gamma^{2}(t)+2 a(t) b(t) \gamma(t)+4 a(t) c(t)-b^{2}(t)}{4 a(t)}\right] \\
& \leq \frac{(x(t)-\gamma(t))^{2}}{2 a(t)}\left[\frac{12 a^{2}(t)\left(4 a(t) c(t)-b^{2}(t)\right)-4 a^{2}(t) b^{2}(t)}{12 a^{2}(t)}\right]=\frac{\left.2\left(3 a(t) c(t)-b^{2}(t)\right)\right)}{3 a(t)}(x(t)-\gamma(t))^{2}
\end{aligned}
$$

By $\left(H_{2}\right)$, there is a positive number $\delta$ such that $3 a(t) c(t)-$ $b^{2}(t) \geq \delta>0$, and hence we have

$$
\frac{d V(t, x(t))}{d t} \leq \frac{2 \delta}{3 a_{L}}(x(t)-\gamma(t))^{2} .
$$

Therefore, the periodic solution $\gamma(t)$ of (1) is uniformly asymptotic stable.
Theorem 7. Consider (1); $a(t), b(t), c(t), d(t)$ are all $\omega$-periodic continuous functions; suppose that the following conditions hold:

$$
\begin{aligned}
& \left(H_{1}\right) \quad a(t)>0 \\
& \left(H_{2}\right) \quad b^{2}(t)-3 a(t) c(t)<0 \\
& \left(H_{3}\right) \quad 2 a_{M}|d|_{M}^{2}+\alpha|b|_{M}|d|_{M}-\alpha^{3}<0,
\end{aligned}
$$

where $\alpha=\left(\left(4 a c-b^{2}\right) / 4 a\right)_{L}$; then 
(1) for (1) there exists a unique $\omega$-periodic continuous solution $\gamma(t)$, and

$$
|\gamma(t)| \leq \frac{|d|_{M}}{\alpha}
$$

(2) $\gamma(t)$ is unstable.

Proof. (1) Define a set

$$
\begin{aligned}
B & =\{\varphi(t)|\varphi \in C(R, R), \varphi(t+\omega)=\varphi(t),| \varphi(t) \mid \\
& \left.\leq \frac{|d|_{M}}{\alpha}\right\} ;
\end{aligned}
$$

given any $\varphi(t) \in B$, the norm is defined as follows:

$$
\|\varphi\|=\sup _{t \in[0, \omega]}|\varphi(t)|
$$

thus $(B,\|\cdot\|)$ is a Banach space. Consider the following equation:

$$
\frac{d x}{d t}=\left[a(t) \varphi^{2}(t)+b(t) \varphi(t)+c(t)\right] x+d(t)
$$

By $\left(H_{1}\right)$ and $\left(H_{2}\right)$, we get that

$$
b^{2}(t)-4 a(t) c(t)<0
$$

and

$$
\begin{aligned}
a(t) & \varphi^{2}(t)+b(t) \varphi(t)+c(t) \geq \frac{4 a(t) c(t)-b^{2}(t)}{4 a(t)} \\
& \geq\left(\frac{4 a c-b^{2}}{4 a}\right)_{L}=\alpha>0 .
\end{aligned}
$$

by Lemma $1,(60)$ has a unique $\omega$-periodic continuous solution as follows:

$$
\eta(t)=-\int_{t}^{+\infty} e^{\int_{s}^{t}\left[a(\tau) \varphi^{2}(\tau)+b(\tau) \varphi(\tau)+c(\tau)\right] d \tau} d(s) d s
$$

and

$$
\begin{aligned}
|\eta(t)| & \leq \int_{t}^{+\infty} e^{-\alpha(s-t)}|d(s)| d s \leq \int_{t}^{+\infty} e^{-\alpha(s-t)}|d|_{M} d s \\
& =\frac{|d|_{M}}{\alpha} .
\end{aligned}
$$

Define a map as follows:

$$
\begin{aligned}
(T \varphi)(t) & =\eta(t) \\
& =-\int_{t}^{+\infty} e^{\int_{s}^{t}\left[a(\tau) \varphi^{2}(\tau)+b(\tau) \varphi(\tau)+c(\tau)\right] d \tau} d(s) d s
\end{aligned}
$$

thus if given any $\varphi(t) \in B$, then $(T \varphi)(t) \in B$; hence $T: B \longrightarrow$ $B$; given any $\varphi(t), \psi(t) \in B$, we have

$$
\begin{aligned}
|(T \varphi)(t)-(T \psi)(t)| & =\left|\int_{t}^{+\infty}\left\{e^{\int_{s}^{t}\left[a(\tau) \psi^{2}(\tau)+b(\tau) \psi(\tau)+c(\tau)\right] d \tau}-e^{\int_{s}^{t}\left[a(\tau) \varphi^{2}(\tau)+b(\tau) \varphi(\tau)+c(\tau)\right] d \tau}\right\} d(s) d s\right| \\
& =\left|\int_{t}^{+\infty} e^{\xi} \int_{s}^{t}\left\{a(\tau)\left[\psi^{2}(\tau)-\varphi^{2}(\tau)\right]+b(\tau)[\psi(\tau)-\varphi(\tau)]\right\} d \tau d(s) d s\right| \\
& =\left|\int_{t}^{+\infty} e^{\xi} \int_{s}^{t}[a(\tau)(\varphi(\tau)+\psi(\tau))+b(\tau)][\psi(\tau)-\varphi(\tau)] d \tau d(s) d s\right|
\end{aligned}
$$

where $\xi$ is between $\int_{s}^{t}\left[a(\tau) \varphi^{2}(\tau)+b(\tau) \varphi(\tau)+c(\tau)\right] d \tau$ and $\int_{s}^{t}\left[a(\tau) \psi^{2}(\tau)+b(\tau) \psi(\tau)+c(\tau)\right] d \tau$; thus

$\xi \leq-\alpha(s-t)$, so we have

$$
\begin{aligned}
& |(T \varphi)(t)-(T \psi)(t)| \leq \int_{t}^{+\infty} e^{-\alpha(s-t)} \\
& \cdot \int_{s}^{t}|a(\tau)(\varphi(\tau)+\psi(\tau))+b(\tau)| d \tau|d(s)| d s \\
& \cdot\|\varphi-\psi\| \leq \int_{t}^{+\infty} e^{-\alpha(s-t)} \\
& \cdot \int_{s}^{t}\left(2|a|_{M}|\varphi|_{M}+|b|_{M}\right) d \tau|d|_{M} d s\|\varphi-\psi\| \\
& =\int_{t}^{+\infty} e^{-\alpha(s-t)}\left(2 a_{M}|\varphi|_{M}+|b|_{M}\right)(t-s)|d|_{M} d s
\end{aligned}
$$

$$
\begin{aligned}
& \cdot\|\varphi-\psi\|=\frac{\left(2 a_{M}|\varphi|_{M}+|b|_{M}\right)|d|_{M}}{\alpha^{2}}\|\varphi-\psi\| \\
& =\frac{\left(2 a_{M}\left(|d|_{M} / \alpha\right)+|b|_{M}\right)|d|_{M}}{\alpha^{2}}\|\varphi-\psi\| ;
\end{aligned}
$$

therefore,

$$
\|T \varphi-T \psi\| \leq \frac{\left(2 a_{M}\left(|d|_{M} / \alpha\right)+|b|_{M}\right)|d|_{M}}{\alpha^{2}}\|\varphi-\psi\| .
$$

By $\left(H_{3}\right)$, it follows that

$$
0<\frac{\left(2 a_{M}|d|_{M}+\alpha|b|_{M}\right)|d|_{M}}{\alpha^{3}}<1
$$

by (68) and (69), $T$ is a compression mapping; according to the compression mapping principle, $T$ has a fixed point on $B$, 
the fixed point is the unique $\omega$-periodic continuous solution $\gamma(t)$ of $(1)$, and $|\gamma(t)| \leq|d|_{M} / \alpha$.

(2) Define a Lyapunov function as follows:

$$
V(t, x(t))=(x(t)-\gamma(t))^{2} ;
$$

$$
\begin{aligned}
\frac{d V(t, x(t))}{d t} & =2(x(t)-\gamma(t))\left[\frac{d x(t)}{d t}-\frac{d \gamma(t)}{d t}\right] \\
& =2(x(t)-\gamma(t))\left[a(t)\left(x^{3}(t)-\gamma^{3}(t)\right)+b(t)\left(x^{2}(t)-\gamma^{2}(t)\right)+c(t)(x(t)-\gamma(t))\right] \\
& =2(x(t)-\gamma(t))^{2}\left[a(t)\left(x^{2}(t)+x(t) \gamma(t)+\gamma^{2}(t)\right)+b(t)(x(t)+\gamma(t))+c(t)\right] \\
& =2(x(t)-\gamma(t))^{2}\left[a(t) x^{2}(t)+(a(t) \gamma(t)+b(t)) x(t)+a(t) \gamma^{2}(t)+b(t) \gamma(t)+c(t)\right] \\
& \geq 2(x(t)-\gamma(t))^{2}\left[\frac{4 a(t)\left(a(t) \gamma^{2}(t)+b(t) \gamma(t)+c(t)\right)-(a(t) \gamma(t)+b(t))^{2}}{4 a(t)}\right] \\
& =2(x(t)-\gamma(t))^{2}\left[\frac{3 a^{2}(t) \gamma^{2}(t)+2 a(t) b(t) \gamma(t)+4 a(t) c(t)-b^{2}(t)}{4 a(t)}\right] \\
& \frac{(x(t)-\gamma(t))^{2}}{2 a(t)}\left[\frac{12 a^{2}(t)\left(4 a(t) c(t)-b^{2}(t)\right)-4 a^{2}(t) b^{2}(t)}{12 a^{2}(t)}\right]=\frac{\left.2\left(3 a(t) c(t)-b^{2}(t)\right)\right)}{3 a(t)}(x(t)-\gamma(t))^{2}
\end{aligned}
$$

By $\left(H_{2}\right)$, there is a positive number $\delta$ such that $3 a(t) c(t)-$ $b^{2}(t) \geq \delta>0$, and hence we have

$$
\frac{d V(t, x(t))}{d t} \geq \frac{2 \delta}{3 a_{M}}(x(t)-\gamma(t))^{2} .
$$

Therefore, the periodic solution $\gamma(t)$ of (1) is unstable.

\section{Examples}

The following examples show the feasibility of our main results.

Example 1. Consider the following equation:

$$
\frac{d x}{d t}=(-10+2 \sin x+\sin t) x+4+2 \cos x+\cos t
$$

It is easy to calculate that $\alpha=7, L_{1}=2, L_{2}=$ $2, \sup _{t \in[0,2 \pi]} g(t, 0)=7$, and

$$
\begin{aligned}
|f(t, x)-f(t, y)| & \leq 2|x-y|, \\
|g(t, x)-g(t, y)| & \leq 2|x-y|, \\
L_{2} & =2<\alpha ; \\
\frac{\alpha L_{2}+L_{1} \sup _{t \in[0, \omega]}|g(t, 0)|-L_{2}^{2}}{\alpha\left(\alpha-L_{2}\right)} & =\frac{24}{35}<1 .
\end{aligned}
$$

Clearly, conditions $\left(\mathrm{H}_{1}\right),\left(\mathrm{H}_{2}\right),\left(\mathrm{H}_{3}\right)$ of Theorem 2 are satisfied. It follows from Theorem 2 that system (73) has a unique
$2 \pi$-periodic solution $\gamma(t)$, which is uniformly asymptotic stable. Clearly, by the graph of solution curve, given any initial value $x(0)=x_{0}$ (e.g., $\left.x(0)=0\right)$, the solution curve of $(73)$ tends to the curve of the periodic solution $\gamma(t)$ (see Figure 1).

Example 2. Consider the following equation:

$$
\begin{aligned}
\frac{d x}{d t}= & (-2+\sin t) x^{3}+(1+\cos t) x^{2}-(8+\cos t) x \\
& +2+\sin t
\end{aligned}
$$

It is easy to calculate that $b^{2}(t)-3 a(t) c(t)<0, \alpha=\left(\left(b^{2}-\right.\right.$ $4 a c) / 4 a)_{L}=7, a_{L}=-3,|d|_{M}=3,|b|_{M}=2$, and

$$
-2 a_{L}|d|_{M}^{2}+\alpha|b|_{M}|d|_{M}-\alpha^{3}=-247<0 .
$$

Clearly, conditions $\left(H_{1}\right),\left(H_{2}\right),\left(H_{3}\right)$ of Theorem 6 are satisfied. It follows from Theorem 6 that (75) has a unique $2 \pi$-periodic solution $\gamma(t)$, which is uniformly asymptotic stable.

Clearly, by the graph of solution curve, given any initial value $x(0)=x_{0}($ e.g., $x(0)=0)$, the solution curve of (75) tends to the curve of the periodic solution $\gamma(t)$ (see Figure 2).

\section{Conclusion}

Abel's differential equation plays an important role in many physical and technical applications; because of this, the paper also deals with Abel's differential equation; we first give two results about the existence and uniqueness of the periodic 


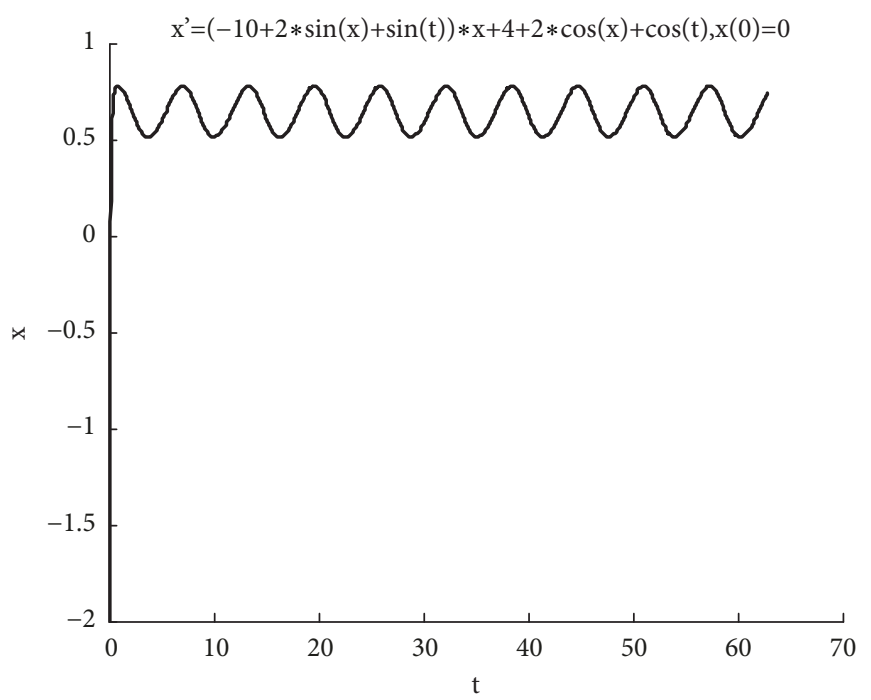

FIGURE 1: The solution curve of (73) with initial value $x(0)=0$.

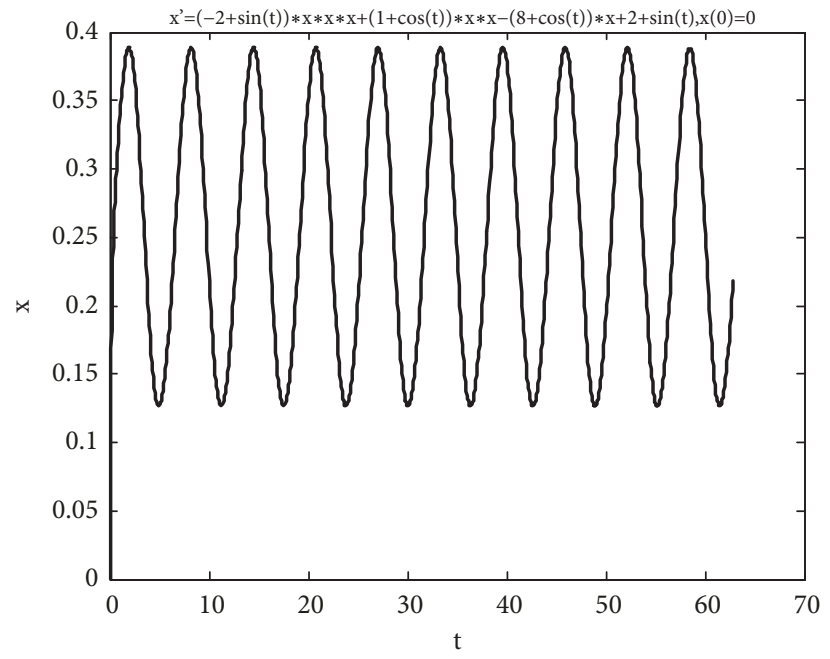

FIgURE 2: The solution curve of (75) with initial value $x(0)=0$.

solution on a nonlinear differential equation by using the fixed point theory; then, we get the existence of the periodic solution of Abel's differential equation, the results having great application value.

\section{Data Availability}

Data sharing is not applicable to this article as no datasets were generated or analysed during the current study.

\section{Conflicts of Interest}

The author declares that there are no conflicts of interest.

\section{Acknowledgments}

This work is supported by special project supported by senior personnel of Jiangsu University (14JDG176).

\section{References}

[1] J. M. Lebrun, On Two Coupled Abel-Type Differential Equations Arising in A Magnetostatic Problem, vol. 103, II Nuovo Cimento, 1990.

[2] M. K. Mak and T. Harko, "Exact causal viscous cosmologies," General Relativity and Gravitation, vol. 30, no. 8, pp. 1171-1186, 1998.

[3] Y. Matsuno, "Two-dimensional dynamical system associated with Abel's nonlinear differential equation," Journal of Mathematical Physics, vol. 33, no. 1, pp. 412-421, 1992.

[4] G. L. Strobel and J. L. Reid, "Nonlinear superposition rule for Abel's equation," Physics Letters A, vol. 91, no. 5, pp. 209-210, 1982.

[5] J. L. Reid and G. L. Strobel, "The nonlinear superposition theorem of Lie and Abel's differential equations," Lettere al Nuovo Cimento. Rivista Internazionale della Società Italiana di Fisica. Serie 2, vol. 38, no. 13, pp. 448-452, 1983. 
[6] M. K. Mak, H. W. Chan, and T. Harko, "Solutions generating technique for Abel-type nonlinear ordinary differential equations," Computers \& Mathematics with Applications, vol. 41, no. 10-11, pp. 1395-1401, 2001.

[7] M. K. Mak and T. Harko, "New method for generating general solution of Abel differential equation," Computers \& Mathematics with Applications, vol. 43, no. 1-2, pp. 91-94, 2002.

[8] A. Cima, A. Gasull, and F. Mañosas, "On the number of polynomial solutions of Bernoulli and Abel polynomial differential equations," Journal of Differential Equations, vol. 263, no. 11, pp. 7099-7122, 2017.

[9] J. Ginï and C. Valls, "Nondegenerate centers for Abel polynomial differential equations of second kind," Journal of Computational and Applied Mathematics, vol. 321, pp. 469-477, 2017.

[10] J. Huang and H. Liang, "Estimate for the number of limit cycles of Abel equation via a geometric criterion on three curves," Nonlinear Differential Equations and Applications NoDEA, vol. 24, no. 4, Art. 47, 31 pages, 2017.

[11] B. Bilbil and M. Sezer, "A numerical approach for solving generalized Abel-type nonlinear differential equations," Applied Mathematics and Computation, vol. 262, no. 1, pp. 169-177, 2015.

[12] H. Ni, L. Tian, and H. Zhang, "The existence and stability of the periodic solutions on Abel differential equation," Mathematica Applicata, vol. 25, no. 4, pp. 854-862, 2012. 


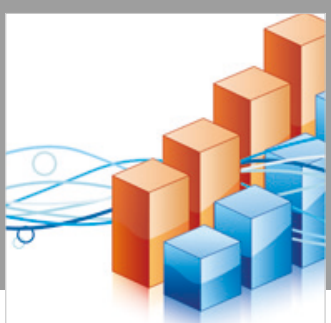

Advances in

Operations Research

\section{-n-m}
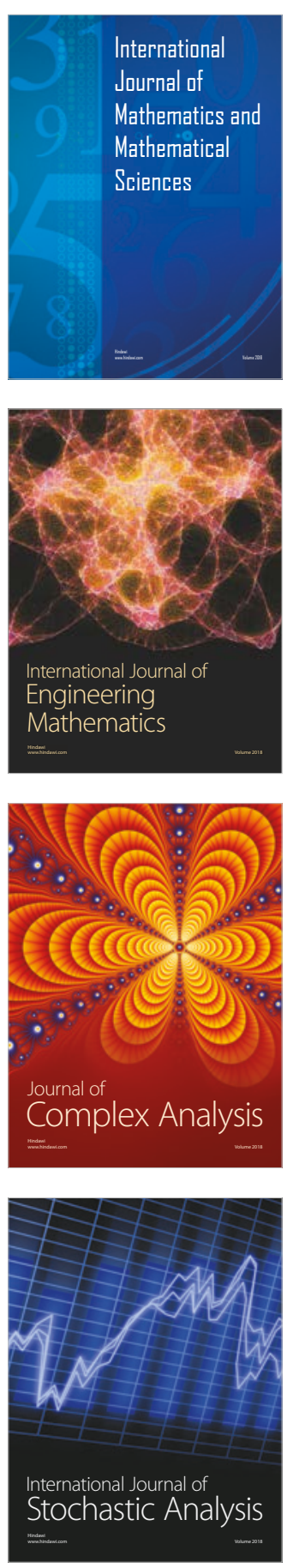
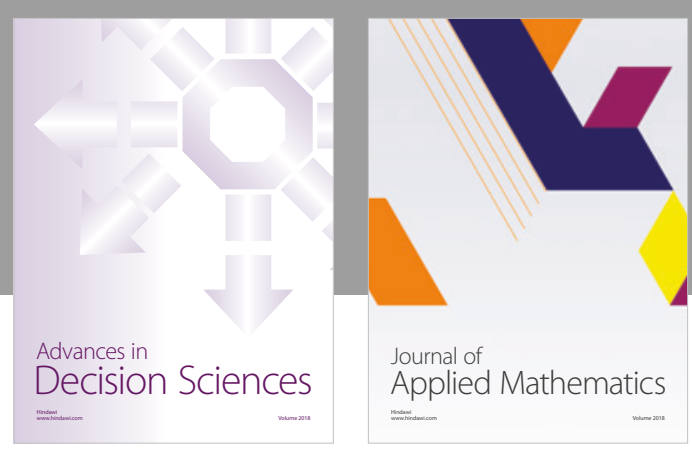

Journal of

Applied Mathematics
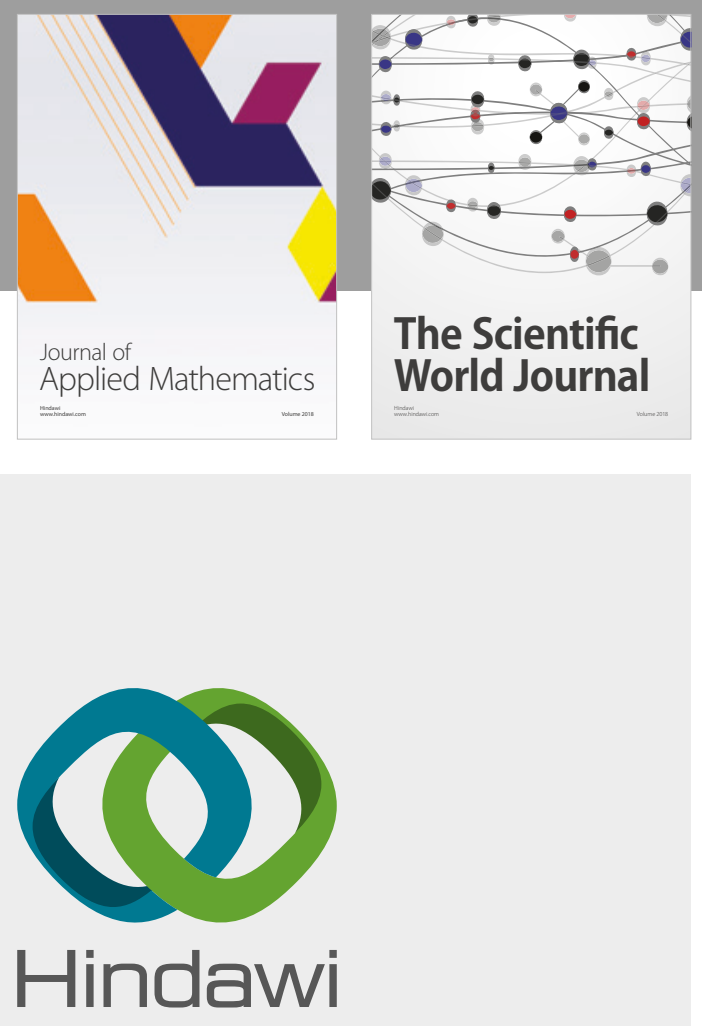

Submit your manuscripts at

www.hindawi.com

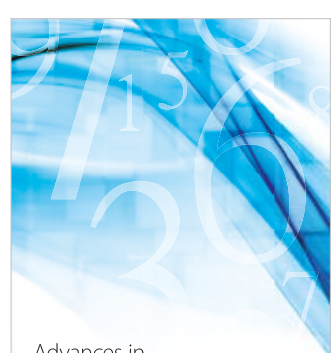

Advances in
Numerical Analysis
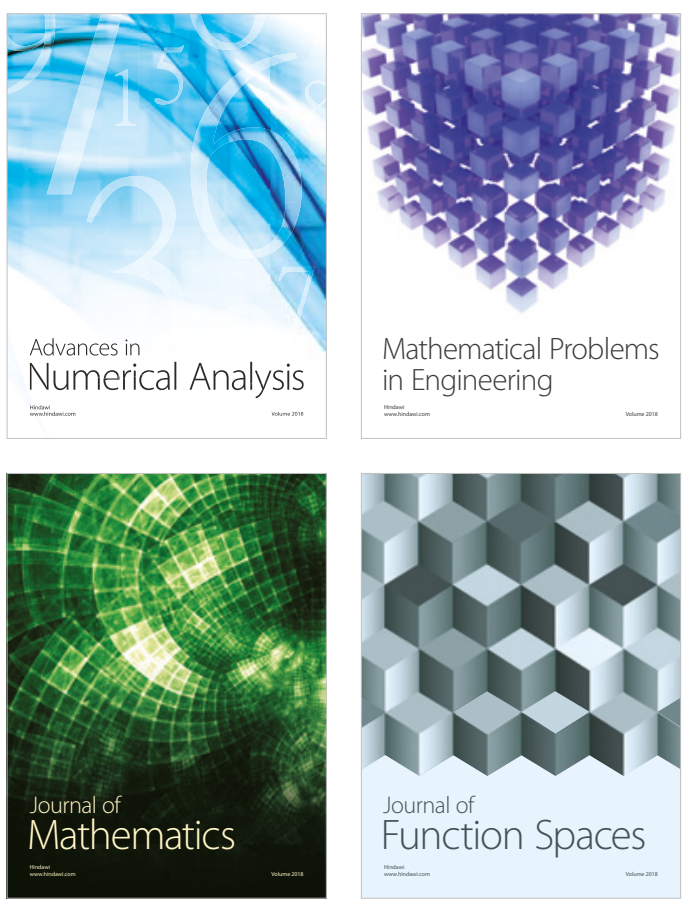

Mathematical Problems in Engineering

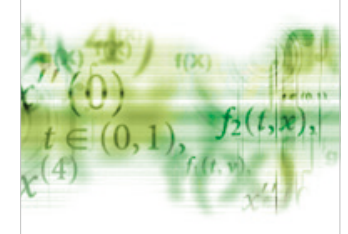

International Journal of

Differential Equations

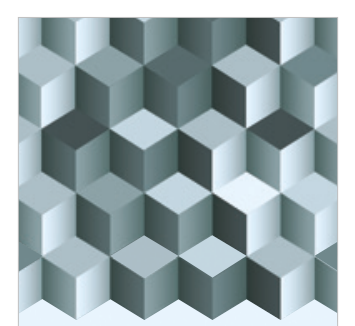

Journal of

Function Spaces

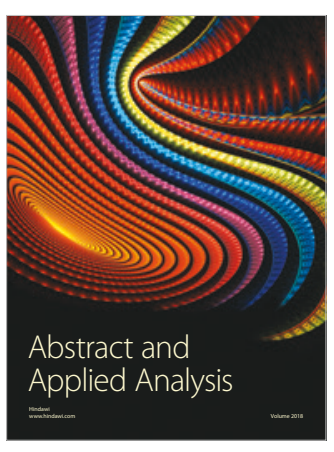

The Scientific

World Journal

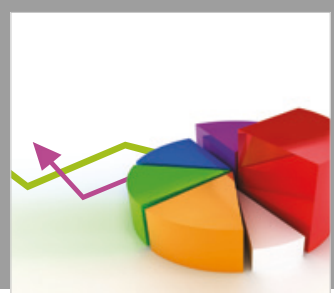

Journal of

Probability and Statistics
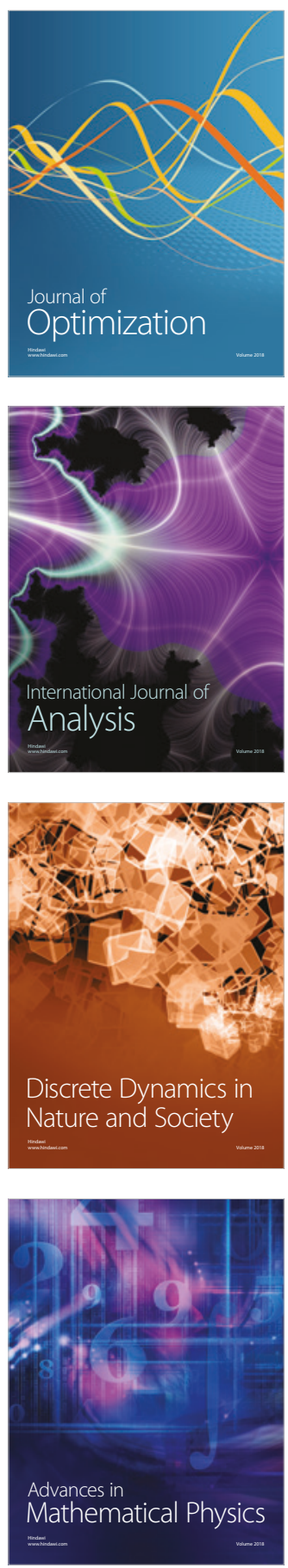